\title{
Recipes for enhanced molecular cooling
}

\author{
Marco Evangelisti* and Euan K. Brechin ${ }^{\dagger}$
}

January 26, 2010

\begin{abstract}
Molecular nanomagnets are considered valid candidates for magnetic refrigeration at low temperatures. Designing these materials for enhanced cooling requires the control and optimization of the quantum properties at the molecular level, in particular: spin ground state, magnetic anisotropy, and presence of low-lying excited spin states. Herein, we present the theoretical framework together with a critical review of recent results, and perspectives for future developments.
\end{abstract}

\section{Introduction}

The Magneto-Caloric Effect (MCE) is based on the change of the magnetic entropy upon application of a magnetic field. All magnetic materials intrinsically show MCE, although the intensity of the effect depends on the properties of each material. Besides the fundamental interest on related thermodynamical properties of novel materials, MCE is of great technological importance since it can be used for cooling applications ${ }^{1}$ according to a process known as adiabatic demagnetization. ${ }^{2}$ This energy-efficient and environmentally friendly technique is particularly promising for refrigeration in the ultra-low-temperature region, providing, e.g., a valid alternative to the use of helium-3 which is quickly becoming rare and expensive. ${ }^{3}$

\footnotetext{
*Instituto de Ciencia de Materiales de Aragón, CSIC-Universidad de Zaragoza, Departamento de Física de la Materia Condensada, 50009 Zaragoza, Spain, E-mail: evange@unizar .es

${ }^{\dagger}$ School of Chemistry, The University of Edinburgh, West Mains Road, EH9 3JJ Edinburgh, United Kingdom
} 
The topic of magnetic refrigeration constitutes one of the potential applications envisioned for molecule-based materials. ${ }^{4}$ Recent studies have demonstrated that the MCE of selected molecular nanomagnets can be much larger than in the best intermetallic and lanthanide alloys, and magnetic nanoparticles, conventionally studied and employed for low-temperature cooling applications. This undoubtedly represents an exciting prospect for these systems, maintaining them in the forefront of investigation in the context of nanoscience and nanotechnology. However in order to be competitive, the molecular magnets need first to be properly designed because characteristics like anisotropy and magnetic interactions among others, set the performance of these materials as coolers. The aim of the present work is to address the physical principles and synthetic strategies which can lead to an enhanced MCE, as well as to provide a brief overview of current activities in this field.

\section{Magneto-caloric effect and refrigeration}

In order to explain the origin of the magneto-caloric effect, we use thermodynamics which relates the magnetic variables (magnetization $M$ and magnetic field $H$ ) to entropy $S_{\mathrm{E}}$ and temperature $T$. Let us recall ${ }^{5}$ that the definition of the entropy of a system having $\Omega$ accessible (and non-degenerate) states is $S_{\mathrm{E}}=k_{\mathrm{B}} \ln (\Omega)$. Since a magnetic moment of spin $S$ has $2 S+1$ magnetic levels, the entropy content per mole of substance associated with the magnetic degrees of freedom between $T=0$ and $T=\infty$ becomes

$$
S_{\mathrm{m}}=R \ln (2 S+1),
$$

where $R=N_{\mathrm{A}} k_{\mathrm{B}}$ is the gas constant. The spin $S$ should be considered as an effective spin describing the multiplicity of the states taking part in the magnetic process.

When a material is magnetized by the application of a magnetic field, the magnetic entropy is changed as the field changes the magnetic order of the material. The MCE and the associated principle of adiabatic demagnetization is readily understood looking at Figure 1. The system, assumed to be a paramagnetic material, is initially in state $A\left(T_{\mathrm{i}}, H_{\mathrm{i}}\right)$, at temperature $T_{\mathrm{i}}$ and field $H_{\mathrm{i}}$. Under adiabatic conditions (i.e., when the 


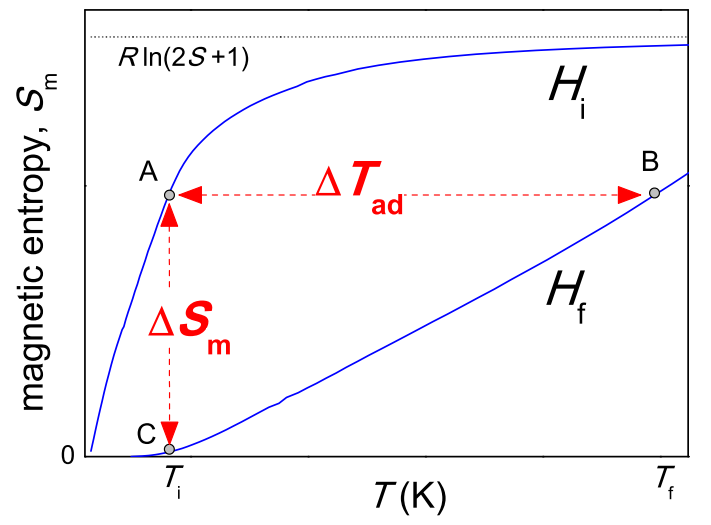

Figure 1: Molar magnetic entropy of a (super)paramagnet with spin $S$ per formula unit, as a function of temperature, for magnetic field $H_{\mathrm{i}}$ and $H_{\mathrm{f}}>H_{\mathrm{i}}$. AB process: adiabatic magnetization providing $\Delta T_{\mathrm{ad}}$. AC process: isothermal magnetization providing $\Delta S_{\mathrm{m}}$.

total entropy of the system remains constant during the magnetic field change), the magnetic entropy change must be compensated for by an equal but opposite change of the entropy associated with the lattice, resulting in a change in temperature of the material. That is, the adiabatic field change $H_{\mathrm{i}} \rightarrow H_{\mathrm{f}}$ brings the system to state $B\left(T_{\mathrm{f}}, H_{\mathrm{f}}\right)$ with the temperature change $\Delta T_{\mathrm{ad}}=T_{\mathrm{f}}-T_{\mathrm{i}}$ (horizontal arrow in Fig. 1). If otherwise the magnetic field is isothermally changed to $H_{\mathrm{f}}$ in a reversible process, the system goes to state $C\left(T_{\mathrm{i}}, H_{\mathrm{f}}\right)$ with the magnetic entropy change $\Delta S_{\mathrm{m}}$ (vertical arrow in Fig. 1). Both $\Delta S_{\mathrm{m}}$ and $\Delta T_{\mathrm{ad}}$ represent the characteristic parameters of the MCE. It is easy to see that if the magnetic change $\Delta H$ reduces the entropy $\left(\Delta S_{\mathrm{m}}<0\right)$, then $\Delta T_{\text {ad }}$ is positive, whereas if $\Delta H$ is such that $\Delta S_{\mathrm{m}}>0$, then $\Delta T_{\mathrm{ad}}<0$ (Fig. 1). 


\section{Evaluation of MCE from magnetization and heat ca- pacity experiments}

In order to establish the relationship between $H, M$ and $T$ to the MCE terms, $\Delta T_{\mathrm{ad}}$ and $\Delta S_{\mathrm{m}}$, we consider the Maxwell equation for the magnetic entropy

$$
\left(\frac{\partial S_{\mathrm{m}}(T, H)}{\partial H}\right)_{T}=\left(\frac{\partial M(T, H)}{\partial T}\right)_{H} .
$$

Integrating Eq. (2) for an isothermal process, we obtain

$$
\Delta S_{\mathrm{m}}(T, \Delta H)=\int_{H_{\mathrm{i}}}^{H_{\mathrm{f}}}\left(\frac{\partial M(T, H)}{\partial T}\right)_{H} \mathrm{~d} H .
$$

This equation indicates that $\Delta S_{\mathrm{m}}$ is proportional to both the derivative of magnetization with respect to temperature at constant field and to the field variation. The accuracy of $\Delta S_{\mathrm{m}}$ calculated from magnetization experiments using Eq. (3) depends on the accuracy of the measurements of the magnetic moment, $T$ and $H$. It is also affected by the fact that the exact differentials are replaced by the measured variations $(\Delta M, \Delta T$ and $\Delta H)$.

Using the following thermodynamic relations:

$$
\begin{aligned}
\left(\frac{\partial T}{\partial H}\right)_{S_{\mathrm{m}}} & =-\left(\frac{\partial S_{\mathrm{m}}}{\partial H}\right)_{T}\left(\frac{\partial T}{\partial S_{\mathrm{m}}}\right)_{H}, \\
C & =T\left(\frac{\partial S_{\mathrm{m}}}{\partial T}\right)_{H},
\end{aligned}
$$

where $C$ is the heat capacity at constant field, and taking into account Eq. (2), the adiabatic temperature change is given by

$$
\Delta T_{\mathrm{ad}}(T, \Delta H)=\int_{H_{\mathrm{i}}}^{H_{\mathrm{f}}}\left(\frac{T}{C(T, H)}\right)_{H}\left(\frac{\partial M(T, H)}{\partial T}\right)_{H} \mathrm{~d} H .
$$

The measurement of the heat capacity as a function of temperature in constant magnetic field provides the most complete characterization of MCE in magnetic materials. From the experimental heat capacity, the temperature dependence of the magnetic entropy 
$S_{\mathrm{m}}(T)$ is obtained by integration, i.e., using

$$
S_{\mathrm{m}}(T)=\int_{0}^{T} \frac{C_{\mathrm{m}}(T)}{T} \mathrm{~d} T
$$

where $C_{\mathrm{m}}(T)$ is the magnetic heat capacity as obtained by subtracting the lattice contribution to the total $C$ measured. Hence, if $S_{\mathrm{m}}(T)$ is known for $H_{\mathrm{i}}$ and $H_{\mathrm{f}}$, both $\Delta T_{\mathrm{ad}}(T, \Delta H)$ and $\Delta S_{\mathrm{m}}(T, \Delta H)$ can be obtained. The accuracy in the evaluation of MCE using heat capacity data depends critically on the accuracy of the $C$ measurements and data processing (e.g., use of $\Delta T$ instead of $\mathrm{d} T$ ). An additional source of uncertainty may eventually be given by the fact that, in order to carry out the integration of Eq. (5), one has to extrapolate the experimental magnetothermal data to $T=0$ and to $T=\infty$.

\section{Advantages of molecular magnets for MCE}

Nanomagnets were proposed for magnetic refrigeration by Shull and co-workers ${ }^{6}$ in the 1990s. Nanoclustering the spin moments into non-interacting particles results in a net magnetic moment per particle, which is typically large and therefore yields large entropy according to Eq. (1). Superparamagnetic nanoparticles composed of rare-earth and transition-metal ions have attracted much the recent interest, ${ }^{7,8}$ but the presence of non-active solvent, interparticle interactions and particle size distributions, are all ingredients that negatively affect the performance of these nanostructured materials in terms of the MCE. Molecular magnets are in many ways superior to magnetic nanoparticles, because they exhibit ideal mono-dispersity in size, shape and magnetic moment. Furthermore, their molecular character opens up avenues for a fine tuning of their magnetic properties. As will be exemplified below, this last point is crucial for improving the performance in terms of MCE.

\subsection{Molecular anisotropy}

The splitting of the molecular energy levels gives rise to a characteristic heat capacity behaviour, that is the Schottky anomaly. Given the electronic configuration and so 
the partition function $Z$ of a system, the calculation of its Schottky contribution is straightforward by using the thermodynamic relationship

$$
C=\frac{\partial}{\partial T}\left(R T^{2} \frac{\partial \ln Z}{\partial T}\right) .
$$

The general formula of the Schottky anomaly for a set of energy levels $E_{i}$ and corresponding degeneracies $g_{i}$ is given by

$$
C_{\mathrm{Sch}}=\left(\frac{1}{k_{\mathrm{B}} T}\right)^{2} \frac{\sum_{i, j} g_{i} g_{j}\left(E_{i}^{2}-E_{i} E_{j}\right) \exp \left[-\left(E_{i}+E_{j}\right) / k_{\mathrm{B}} T\right]}{\sum_{i, j} g_{i} g_{j} \exp \left[-\left(E_{i}+E_{j}\right) / k_{\mathrm{B}} T\right]} .
$$

In a superparamagnetic molecular cluster (a.k.a. single-molecule magnet), the presence of an anisotropy barrier gives rise to a Schottky anomaly. ${ }^{9}$ The barrier is, indeed, the result of zero-field splitting brought about by crystal-field effects arising from the metal oxidation states and surrounding organic ligands, and sets a preferential direction for the molecular spins. The picture depicted in Figure 1 is still valid for a superparamagnet with $S$ as net cluster moment, provided that it is at temperatures above the blocking temperature. The molecular magnetic anisotropy, which determines the blocking temperature, can be considered as a drawback in the MCE efficiency of superparamagnets. Let us demonstrate this last statement with the following example.

Consider three systems of non-interacting monodisperse magnetic clusters with spin $S=10$ and hypothetical axial anisotropies $D=-0.5,-1.5$ and $-3.0 \mathrm{~K}$, respectively. We first calculate the corresponding Schottky heat capacities $C_{\text {Sch }}$ according to Eq. (6) obtaining results akin to the ones depicted in the top panel of Figure 2 for the case of $D=-0.5 \mathrm{~K}$ and different applied fields. From these, we calculate the corresponding magnetic entropies $S_{\mathrm{m}}(T, H)$ by using Eq. (5). Finally, by knowing $S_{\mathrm{m}}(T, H)$ we obtain the magnetic entropy changes $\Delta S_{\mathrm{m}}(T, \Delta H)=\left[S_{\mathrm{m}}\left(T, H_{\mathrm{f}}\right)-S_{\mathrm{m}}\left(T, H_{\mathrm{i}}\right)\right]$ for the given field change of $\Delta H=(70-10) \mathrm{kG}$. The results are depicted in the bottom panel of Fig. 2, where it can be noticed that the increase of the anisotropy tends to lower the values of $-\Delta S_{\mathrm{m}}$ (and similarly $\Delta T_{\mathrm{ad}}$ ) by shifting them towards higher temperatures. In other words, if the anisotropy is large, the polarization of the molecular spins is less sensitive to $H$, or (equivalently) higher fields are needed, thus yielding a lower MCE. 


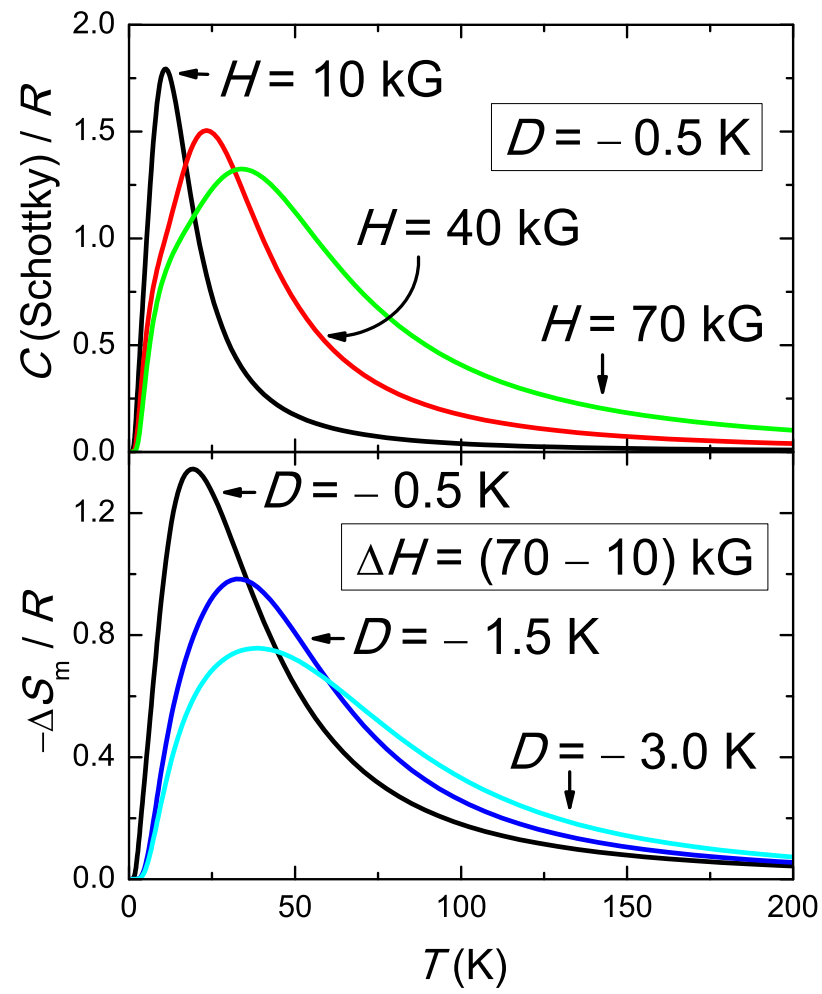

Figure 2: Top: Schottky heat capacities $C_{\text {Sch }}$ of an isolated magnetic particle with $S=10$ and axial anisotropy $D=-0.5 \mathrm{~K}$ calculated for $H=10,40$ and $70 \mathrm{kG}$, respectively. Bottom: Magnetic entropy changes $\Delta S_{\mathrm{m}}$ obtained from the top panel data and equivalent systems with $D=-1.0$ and $3.0 \mathrm{~K}$ following an applied field change of $\Delta H=(70-10) \mathrm{T}$.

Furthermore, it is important to consider that for temperatures below the blocking temperature, the spin-lattice relaxation slows down dramatically, i.e. the molecular spins tend to lose thermal contact with the lattice, as revealed again by heat capacity experiments. ${ }^{10}$ This off-equilibrium results in lower magnetic entropies and, consequently, lower MCE parameters. Therefore, if we target the highest performance as magnetic refrigerants, we should design the molecular magnets in such a way to have a negligible anisotropy which would permit an easy polarization of the net molecular spin, leading to a large magnetic entropy change. The above discussion also demonstrates that, in order to be successful, the applicability of the (isotropic) molecular coolers has to be 

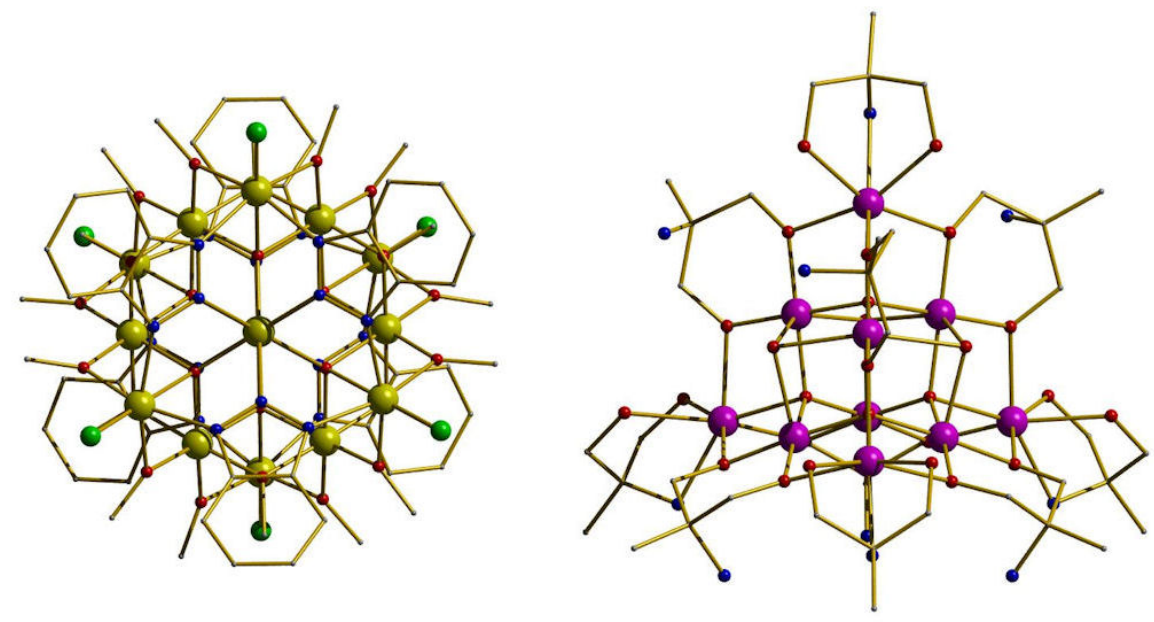

Figure 3: Molecular structures of $\mathrm{Fe}_{14}$ (left) and $\mathrm{Mn}_{10}$ (right). Yellow Fe, purple $\mathrm{Mn}$, green $\mathrm{Cl}$, red $\mathrm{O}$, blue $\mathrm{N}$. Hydrogen atoms are omitted for clarity.

in the very-low-temperature region.

The archetypal highly-anisotropic $\mathrm{Mn}_{12}$ and $\mathrm{Fe}_{8}$ molecular magnets were the first to be investigated for magnetic refrigeration by Tejada and colleagues, ${ }^{11}$ Zhang and colleagues, ${ }^{12}$ and Tishin and colleagues. ${ }^{4}$ Owing to their well-defined spin ground state $S=10$ at low temperatures, it is easy to show using Eq. (1) that $\mathrm{Mn}_{12}$ and $\mathrm{Fe}_{8}$ cannot have values of $-\Delta S_{\mathrm{m}}$ exceeding 12.5 and $11 \mathrm{Jkg}^{-1} \mathrm{~K}^{-1}$, respectively. Although these values are relatively large, they also are difficult to observe because the large anisotropy present in both systems freezes the orientation of the molecular spins once the temperature is lowered below $(2-4) \mathrm{K}$. This limits their applicability as refrigerants. The search for isotropic molecular coolers led physicists in Modena together with chemists in Manchester to the heterometallic wheel $\mathrm{Cr}_{7} \mathrm{Cd},{ }^{13}$ whose limitation is in the low value of the spin $S=3 / 2$. It is only very recently that a huge step forward has been accomplished via the synthesis and study of highly-symmetric molecules with high values of the spin ground state. The first was the $\mathrm{Fe}_{14}$ with a remarkable $S=25$ ground state, ${ }^{14,15}$ and second the supertetrahedron $\mathrm{Mn}_{10}$, a ferromagnetic cluster with $S=22$ displaying practically zero anisotropy (Fig. 3). ${ }^{16}$ This fascinating combination results in MCE responses nearly three times greater than that of $\mathrm{Cr}_{7} \mathrm{Cd}$, and comparable to that of one of the best performing superparamagnetic nanoparticles, nominally 
$\mathrm{DyCo}_{2} \cdot{ }^{8}$

\subsection{Molecular spin degeneracy}

A property favouring large $\mathrm{MCE}$ is the presence of degenerate or low-lying excited spin states, since the so-added degrees of freedom result into an extra magnetic entropy content according to Eq. (1). This situation is encountered, for instance, in magneticallyfrustrated systems, as was recently pointed out by Schnack et al. ${ }^{17}$ and Zhitomirsky, ${ }^{18}$ who concluded that in terms of MCE the field-dependent efficiency of a geometrically frustrated magnet can exceed that of an ideal paramagnet with equivalent spin by more than an order of magnitude. In this regard recent results obtained for the magneticallyfrustrated $\mathrm{Fe}_{14}$ molecular cluster compound are indeed quite promising. ${ }^{15}$

Molecular magnets have recently proved that an alternative and simpler approach to frustration in promoting spin-degeneracy can be obtained by designing very weak magnetic links between the single-ion spin centers. ${ }^{19-21}$ Let us present the physics involved in the way in which intramolecular magnetic ordering into a total spin $S_{\text {tot }}=n s$ of a number $n$ of spins $s$, which are part of the same molecule, can lead to a partial concentration of the total magnetic entropy change into a limited range of temperature. If a molecule is paramagnetic, with $n$ non-interacting spins $s$, the magnetic entropy per mole is $S_{\mathrm{m}}^{(\mathrm{n} \text {-in) }}=n R \ln (2 s+1)$ from Eq. (1). In the low-temperature range, however, where the $n$ spins $s$ couple into $S_{\text {tot }}=n s$, the entropy change to consider is $S_{\mathrm{m}}^{(\text {in })}=R \ln \left(2 S_{\mathrm{tot}}+1\right)=R \ln (2 n s+1)$, which is clearly different. Of course the total magnetic entropy gain that can be reached between zero and infinite temperature

remains equal to $S_{\mathrm{m}}^{(\mathrm{n} \text {-in) }}$, which is the maximum entropy gain. However, the way in which the magnetic entropy is released as a function of temperature is changed by the presence of the interactions from the smooth gradual temperature dependence of $S_{\mathrm{m}}(T)$ in the paramagnetic case, into a dependence showing more steep behaviour in the temperature range where the interactions become important. This can be used advantageously to produce a large entropy change by means of a limited change in temperature and/or field, that is a much larger change than can be produced in the absence of such interactions, yielding an enhanced MCE. 


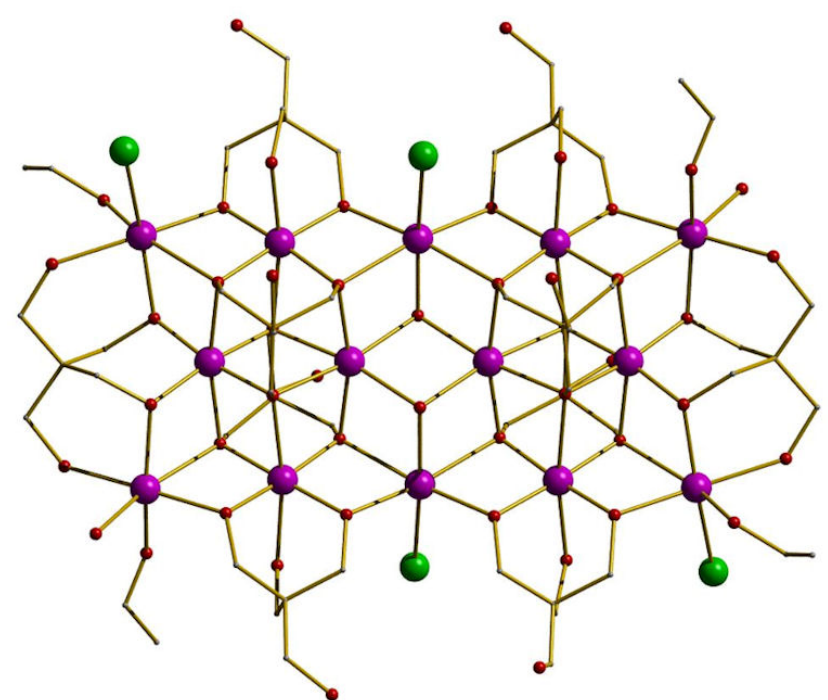

Figure 4: Molecular structures of $\mathrm{Mn}_{14}$. Purple Mn, green I, red O, blue N. Hydrogen atoms are omitted for clarity.

The magnetic ordering in a bulk solid-state materials, e.g. pure gadolinium, is conceptually analogous to the aforementioned case of the cluster molecule. For a system of non-interacting $\mathrm{Gd}$ spins the temperature dependence of the magnetic entropy will show a smooth behaviour, gradually varying from zero at $T=0$ to the maximum value, $R \ln (2 S+1)$ with $S=7 / 2$ for Gd. When introducing the magnetic interactions, the resulting magnetic ordering at $T_{C}=293 \mathrm{~K}$ of the Gd spins will result in a steep fall of the entropy curve below $T_{C}$ to near zero value in the magnetically ordered region. In principle one could then play similar games, i.e. producing large entropy changes by small changes in field or temperature. Unfortunately, the $T_{C}$ value of $\mathrm{Gd}$ metal is too high for any practical purposes (here the magnetic contribution to the total heat capacity is in fact negligible compared to the lattice contribution). It is worth mentioning that the MCE driven by long-range magnetic order at high temperatures has already been investigated in molecule-based materials, nominally Prussian blue analogues. ${ }^{22}$

The successful chemistry approach investigated so far for promoting weaker pairwise intramolecular exchanges has been that of making use of bridging hydroxides, $\mathrm{Mn}^{2+}$ or lanthanide ions. ${ }^{19}$ These strategies resulted in a truly enormous enhancement of the MCE: for instance, values of $-\Delta S_{\mathrm{m}}$ as large as $20 \mathrm{Jkg}^{-1} \mathrm{~K}^{-1}$ for liquid-helium 
temperatures and $\Delta H=60 \mathrm{kG}$ have been reported in the case of the $\mathrm{Mn}_{14}$ molecular magnet (Fig. 4), that is almost a factor of two larger than that of the $\mathrm{DyCo}_{2}$ nanoparticles. $^{8}$

\section{Final remarks and perspectives}

While the concept of magnetic refrigeration has been around for a long time, it is with the recent introduction of molecular magnets that the search for ultra-low-temperature coolers has received a huge improvement. These promising materials are the finest refrigerants ever reported for low-temperature applications, that is in a temperature range of efficiency easily accessible with liquid- ${ }^{4} \mathrm{He}$. The underlying physics together with the chemical modifications made feasible by their molecular character, led us to identify which specific aspects of the synthetic procedure may (positively) influence the final output. For instance, inducing spin-degeneracy is a major requirement for enhancing the performance in terms of the magneto-caloric effect. In this respect, the synthesis of new molecules based on $\mathrm{Gd}^{3+}$ ions, which promote weak intramolecular interactions and which possess the largest isotropic spin $(s=7 / 2)$ of any ion in the periodic table, would be a good idea.

A drawback in the use of molecular refrigerants resides in the low density of these materials. For practical purposes, this translates into a relatively large volume of the cooling apparatus. However, this shortcoming does not limit their applicability. Contrary to conventional bulk refrigerants, in which the main entropy changes are driven by phase transitions, here the effect takes place in the nanoscale range, i.e. at the molecular level. We expect that micron- and submicron-sized devices will soon be fabricated for exploiting the functionality of the molecular coolers. This goal requires the surface grafting of the molecules whilst preserving their magnetic properties, a task which cannot be accomplished if the refrigerant bases its efficiency on cooperative interactions. These mesoscopic devices will then find application as cooling platforms for all those instruments where local refrigeration down to very low temperatures is needed. This can include, although is not limited to, high-resolution X-ray and gamma-ray detectors 
for, e.g., astronomy, materials science, and security instrumentation.

\section{Acknowledgments}

The authors are indebted to M. Affronte, L. J. de Jongh, F. Luis and E. J. L. McInnes for enlightening discussions. This work is partially funded by the Spanish Ministry for Science and Innovation through grants MAT2009-13977-C03 and CSD2007-00010, and in the UK through the generous contributions of The Leverhulme Trust and the EPSRC.

\section{References}

[1] See, e.g., (a) C. Zimm, A. Jastrab, A. Sternberg, V. K. Pecharsky, K. A. Gschneidner Jr., M. Osborne and I. Anderson, Adv. Cryog. Eng., 1998, 43, 1759; (b) V. K. Pecharsky and K. A. Gschneidner Jr., J. Magn. Magn. Mater., 1999, 200, 44; (c) K. A. Gschneidner Jr., A. O. Pecharsky and V. K. Pecharsky, in Cryocoolers II, ed. R. S. Ross Jr., Kluwer Academic/Plenum Press, New York, 2001, p. 433.

[2] Originally described by (a) P. Debye, Ann. Phys., 1926, 81, 1154; (b) W. F. Giauque, J. Am. Chem. Soc., 1927, 49, 1864.

[3] See, e.g., T. Feder, Physics Today, 2009, 62, 21.

[4] Yu. I. Spichkin, A. K. Zvezdin, S. P. Gubin, A. S. Mischenko and A. M. Tishin, J. Phys. D: Appl. Phys., 2001, 34, 1162.

[5] See, e.g., C. Kittel, Introduction to Solid State Physics, John Wiley \& Sons, Inc., 2005.

[6] R. D. McMichael R. D. Shull, L. J. Swartzendruber, L. H. Bennett and R. E. Watson, J. Magn. Magn. Mater., 1992, 111, 29.

[7] P. Poddar, S. Srinath, J. Gass, B. L. V. Prasad and H. Srikanth, J. Phys. Chem. C, 2007, 111, 14060. 
[8] S. Ma, W. B. Cui, D. Li, N. K. Sun, D. Y. Geng, X. Jiang and Z. D. Zhang, Appl. Phys. Lett., 2008, 92, 173113.

[9] See, e.g., M. Evangelisti, F. Luis, L. J. de Jongh and M. Affronte, J. Mater. Chem., 2006, 16, 2534, and references therein.

[10] M. Evangelisti, F. Luis, F. L. Mettes, N. Aliaga, G. Aromí, J. J. Alonso, G. Christou and L. J. de Jongh, Phys. Rev. Lett., 2004, 93, 117202.

[11] (a) F. Torres, J. M. Hernández, X. Bohigas and J. Tejada, Appl. Phys. Lett., 2000, 77, 3248; (b) F. Torres, X. Bohigas, J. M. Hernández and J. Tejada, J. Phys.: Condens. Matter, 2003, 15, L119.

[12] X. X. Zhang, H. L. Wei, Z. Q. Zhang and L. Zhang, Phys. Rev. Lett., 2001, 87, 157203.

[13] M. Affronte, A. Ghirri, S. Carretta, G. Amoretti, S. Piligkos, G. A. Timco and R. E. P. Winpenny, Appl. Phys. Lett., 2004, 84, 3468.

[14] M. Evangelisti, A. Candini, A. Ghirri, M. Affronte, E. K. Brechin and E.J.L. McInnes, Appl. Phys. Lett., 2005, 87, 072504.

[15] R. Shaw, R. H. Laye, L. F. Jones, D. M. Low, C. Talbot-Eeckelaers, Q. Wei, C. J. Milios, S. Teat, M. Helliwell, J. Raftery, M. Evangelisti, M. Affronte, D. Collison, E. K. Brechin and E. J. L. McInnes, Inorg. Chem., 2007, 46, 4968.

[16] M. Manoli, R. D. L. Johnstone, S. Parsons, M. Murrie, M. Affronte, M. Evangelisti and E. K. Brechin, Angew. Chem. Int.-Ed., 2007, 46, 4456.

[17] J. Schnack, R. Schmidt and J. Richter, Phys. Rev. B, 2007, 76, 054413.

[18] M. E. Zhitomirsky, Phys. Rev. B, 2003, 67, 104421.

[19] M. Manoli, A. Collins, S. Parsons, A. Candini, M. Evangelisti and E. K. Brechin, J. Am. Chem. Soc., 2008, 130, 11129.

[20] M. Evangelisti, A. Candini, M. Affronte, E. Pasca, L. J. de Jongh, R. T. W. Scott and E. K. Brechin, Phys. Rev. B, 2009, 79, 104414. 
[21] G. Karotsis, M. Evangelisti, S. J. Dalgarno and E. K. Brechin, Angew. Chem. Int.-Ed., 2009, 48, 9928.

[22] (a) E. Manuel, M. Evangelisti, M. Affronte, M. Okubo, C. Train and M. Verdaguer, Phys. Rev. B, 2006, 73, 172406; (b) M. Evangelisti, E. Manuel, M. Affronte, M. Okubo, C. Train and M. Verdaguer, J. Magn. Magn. Mater., 2007, 316, e569. 